\title{
Protée
}

\section{Des origines de la peinture}

\section{Éric Méchoulan}

Volume 28, numéro 1, 2000

\section{Variations sur l'origine}

URI : https://id.erudit.org/iderudit/030580ar

DOI : https://doi.org/10.7202/030580ar

Aller au sommaire du numéro

Éditeur(s)

Département des arts et lettres - Université du Québec à Chicoutimi

ISSN

0300-3523 (imprimé)

1708-2307 (numérique)

Découvrir la revue

\section{Citer cet article}

Méchoulan, É. (2000). Des origines de la peinture. Protée, 28(1), 19-29. https://doi.org/10.7202/030580ar

\section{Résumé de l'article}

Les discours sur l'origine de la peinture (ceux de Pline l'Ancien, d'Alberti, mais aussi ceux de la théologie chrétienne) permettent de saisir trois manières par où affleurent la source et la production de la peinture : l'ombre, le miroir, l'empreinte. d'utilisation que vous pouvez consulter en ligne.

https://apropos.erudit.org/fr/usagers/politique-dutilisation/ 


\section{DES O RIG INES DE LA PEINTURE}

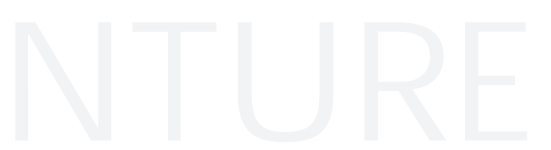

ÉRIC MÉCHOULAN

L'image de l'invisible est elle-même invisible, sinon elle n'en serait pas l'image.

J. Chrysostome, Homélie sur l'épître aux Colossiens.

"La peinture vient s'inscrire sur un fond blanc», ces mots contiennent en germe toute réflexion sur la peinture.

T. Chikuden, Bavardages d'un ermite de la montagne.

\section{AU COMMENCEMENT ÉTAIT L'OMBRE: LA PEAU DU MUR}

L'origine de la peinture n'appartient pas à la peinture. Lorsque Pline l'Ancien, dans sa rêverie encyclopédique, retrace l'histoire de la peinture au livre XXXV de son Histoire naturelle, il n'en dit que ceci:

La question des origines de la peinture est obscure (incerta) et n'entre pas dans le plan de cet ouvrage. Les Égyptiens déclarent qu'elle a été inventée chez eux six mille ans avant de passer en Grèce: vaine prétention, c'est bien évident. Quant aux Grecs, les uns disent que le principe en a été découvert à Sicyone, les autres à Corinthe, et tous reconnaissent qu'il a consisté à tracer, grâce à des lignes, le contour d'une ombre humaine (umbra hominis lineis circumducta). ${ }^{1}$

Pas de mythe d'origine, donc, et pas même de lieu sûr pour son invention, mais, à tout le moins, une technique: ce qui est inauguré est le dessin et non la couleur, la silhouette et non l'expression. Le verbe employé par Pline revêt une certaine importance, car si, littéralement, il désigne bien le fait de conduire la main qui trace autour de l'ombre portée d'un homme la ligne étroite de sa silhouette, il renvoie de manière générale à l'habitude de tracer un cercle avec la charrue, en particulier lors des fondations des villes ${ }^{2}$, mais il peut aussi, en un curieux jeu d'ombres, signifier duper ou escroquer quelqu'un, escamoter un objet à la façon dont les Latins entouraient un objet d'un cercle en guise d'annulation. La naissance de la peinture réside dans cette appropriation de l'ombre qui fonde et annule du même mouvement: d'origine de la peinture, il n'existe qu'une fondation biffée. Elle n'est donc pas simplement incertaine, mais d'elle-même trompeuse et égarante, événement qui n'apparaît que dans le geste par où il 
s'efface. Si l'on veut circonscrire l'origine de la peinture, il faut décidément lâcher la proie pour l'ombre.

Car elle existe bel et bien cette origine et Pline la raconte; mais il ne la raconte pas dans la partie consacrée à la peinture. À peine en a-t-il fini avec celleci qu'il évoque l'origine du modelage et, en douce (presque en taille-douce), la naissance de la peinture:

En voilà assez et plus qu'il n'en faut sur la peinture. Il serait convenable d'y rattacher ce qui concerne le modelage. En utilisant lui aussi la terre, le potier Butadès de Sicyone découvrit le premier l'art de modeler des portraits en argile; cela se passait à Corinthe et il dut son invention à sa fille, qui était amoureuse d'un jeune homme; celui-ci partant pour l'étranger, elle entoura d'une ligne (circumscribit) l'ombre de son visage projetée sur le mur par la lumière d'une lanterne; son père appliqua de l'argile sur l'esquisse, en fit un relief qu'il mit à durcir au feu avec le reste de ses poteries, après l'avoir fait sécher. ${ }^{3}$

Là où l'origine de la peinture fait l'objet de conflits d'attribution - l'Égypte, rapidement éliminée, puis, sans que l'on puisse trancher, Sicyone et Corinthe -, voici maintenant que chacun s'accorde. La naissance en revient à la fille d'un potier de Sicyone, mais établi à Corinthe: les deux traditions se fondent l'une en l'autre.

On admirera le déplacement nécessaire pour que l'origine de la peinture puisse être dite: œuvre féminine, elle a besoin de la médiation d'un homme; ouvrage de jeune fille, elle requiert la puissance d'un père; travail fragile du désir, elle passe par l'industrie de l'expert; platitude d'une ombre qui va disparaitre, il lui faut le relief du moule pour vraiment apparaittre. À peine née, la peinture s'évanouit sous le modelage comme l'ombre sous l'argile (ou encore comme l'homme sous la main toute-puissante du Dieu potier). Cette terre appliquée verticalement sur le dessin de l'ombre est sa tombe plus encore que sa trace. Là où la jeune fille avait soigneusement écrit le tour de son ami, elle voit apparaître une médaille ou une sculpture tombale ${ }^{4}$. «Écrire le tour», tel est le terme latin, circumscribere (plutôt que circumducere, cette fois), qui a plus que son prédécesseur une valeur d'enfermement et de clôture, mais offre lui aussi les valeurs de tromperie et d'élimination. L'ombre trompe l'absence comme on cherche, en suçant un bout d'étoffe, à tromper la faim. La jeune fille circonscrit l'homme qu'elle aime, elle écrit son ombre (les Grecs désignent ainsi le procédé: skiagraphein, qui renvoie aussi aux pratiques de trompe-l'œil et d'illusionnisme pictural). Le travail de l'ombre est un travail de l'illusion, un aveuglement qui illumine.

À l'origine de la peinture se trouverait le désir de la présence, et la présence respire dans l'ombre portée d'un corps. C'est ce que comprend cette jeune fille partagée, moitié de Sicyone, moitié de Corinthe: plutôt que de laisser simplement s'en aller son jeune amant sur les chemins du souvenir, la voici qui dessine, d'un trait de charbon, son ombre sur le mur. Il est désormais prisonnier de cette ombre comme Antigone emmurée vive et protégée par les tablettes d'une loi non écrite. L'écriture de l'ombre est présence vive de la mémoire, manière d'attraper l'événement d'un corps: non tant pour l'emprisonner peut-être que pour mieux se rendre soi-même prisonnière de cet événement.

Fille d'un artisan agile, d'un sculpteur de matières utiles, d'un tourneur d'argile, elle souscrit à la gratuité du désir qui ne trouve sa valeur que dans ses propres projections. La jeune fille du potier inscrit la peinture dans l'ordre du privé: elle n'en fait pas un fétiche public ou une beauté collective. En deçà des devoirs de la polis, par ce geste intime, elle trace un cercle qui l'isole dans l'intimité du geste. Là où le potier, modeleur, cherche des masses soumises à son tour de main, sa fille avance sur une ligne, la plus mince possible afin d'épouser au plus près l'exactitude de la peau. La ligne, voilà l'affaire de la peinture: lorsque Protogène et Apelle rivalisent, c'est à qui dessinera, sur le trait de l'autre, la ligne plus fine encore qui, en le biffant, le dépassera ${ }^{5}$. C'est une ouvre de la limite: limite ligneuse d'un corps, limite aussi de ce qui peut matériellement faire l'objet d'un trait. Mais un trait qui n'enserre plus rien: ce n'était pas même le corps qui se trouvait là, seulement son ombre. Et même l'ombre s'en est allée, mais non sa limite. 
Une ombre est-elle voile jeté sur le corps ou sa plus radicale nudité? L'ombre escroquée au corps de son amant par le tracé de son contour, est-ce la nuit qui fait son trou dans le mur, qui s'y love et s'y thésaurise, ou bien la lecture émouvante d'un front, d'un nez, d'une bouche? Car ce qui s'écrit noir sur blanc s'apprête à être lu. C'est un exercice de mémoire. Les Grecs n'ont pas de nom pour la lecture: ils utilisent les mots du souvenir (anagiskein) et de la reconnaissance (anagnorisis). Le visage tapi dans le mur, la jeune fille peut le reconnaître: non seulement s'agit-il de l'amoureuse silhouette de son amant, mais c'est elle qui l'a écrite. Elle a écrit l'ombre de son amant et, en même temps, sa séparation d'avec lui: le trait sépare autant qu'il unit. Dans la silhouette dessinée sur le mur, elle peut lire la présence d'un profil autant que l'absence d'un corps. Le trait sans relief auquel le père donnera la profondeur de la matière est un signe, un signe d'ombre, un signe qui reste à la surface, faute de pouvoir escamoter le vide. L'aspiration de ce signe d'ombre n'est pas de renvoyer à la plénitude d'une jouissance impeccable, mais de faire s'évanouir le néant que cette jouissance creusait, de libérer le fond de sa présence vaine. C'est un signe qui ne connait rien que la surface - mais n'est-ce pas le destin de tout signe, quand bien même il prétend se superposer aux choses qu'il désigne?

Le modelage, lui, superpose exactement les matières: ce n'est plus un signe, c'est une similitude. La peinture, en sa naissance, est une lecture angulaire. Elle forme une géométrie spéculaire sur la peau rude d'un mur; elle travaille dans l'angle mort du modelage. Le signe d'ombre est désir de signe, le modelage du corps est forme d'un modèle: l'un s'articule à la singularité, l'autre à l'identité. Mais le destin de la peinture est de conserver, de façon intempestive, la puissance du singulier, tout en se faisant recouvrir par le type et le modèle. La lecture, plus encore que l'écriture, est cette expérience du singulier. La peinture naît, bien sûr, du geste de tracer: c'est d'abord une main animée d'un désir de présence (mais une main qui a justement lâché le corps de l'amant pour tenir entre ses doigts le froid morceau de charbon brûlé, qui a déjà renoncé au plaisir de l'instant pour anticiper le frisson de l'absence en la présence même de celui qu'elle désire); et la lecture lui donne le goût de sa passion: c'est un regard rivé à la surface d'un signe. Dans la peinture, nous lisons ce qui nous fonde, la ligne qui nous définit, la chaleur contemplée d'un instant gelé.

S'il s'agit de fonder une ville, le conducteur de la charrue est masculin. Mais ici, le fait d'avoir une jeune fille nous invite à concevoir une autre origine pour la peinture, un autre genre de fondation que la puissance d'éventration, un autre artisanat que la poterie et sa jouissance des creux et des trous: la ligne est, en fait, affaire de tissage, celle qui travaille le lin connaît le mouvement des lignes. Avant d'être trait de plume ou de pinceau, avant même d'être traits du visage, la ligne est d'abord fil de lin, puis cordeau pour monter les murs ou filet pour attraper les poissons. La ligne fait notre linge, elle limite nos chambres, elle piège nos nourritures. Et, finalement, elle enfouit nos corps sous les plis bien lissés d'un linceul. L'écriture est ligne, le visage est ligne: ce sont des vêtements de lin qui disent nos corps. L'ombre portée sur le mur et dessinée d'un trait nous offre donc un voile qui est notre propre nudité, un lin noir qui n'est ni copie, ni modèle: ce sont des vêtements d'ombre qui disent la vérité du nu.

La jeune fille sous le père, voilà ce que révèle l'origine de la peinture ${ }^{6}$. La ligne du lin comme un point de rebroussement dans la courbe du lignage. L'invention du père potier est fille de celle de sa fille peintre. La peinture est l'ombre venant avant le corps et l'origine de la peinture, peinture de la limite.

Mais à quel moment naît cette peinture: dans l'instant où la jeune fille, d'une main attentive, trace le contour de la silhouette aimée ou dans le moment où l'ombre de l'amant a quitté la surface du mur et que la blancheur du fond, tout à coup, apparaît? La naissance est double: d'abord la main et son mouvement, ensuite l'œil et son regard. Il faut le trait qui circonscrive l'ombre, puis ce mince trait noir qui écrit la peau sombre du jeune homme, comme si l'ombre avait pu repousser toute sa noirceur jusqu'à sa 
surface, libérant alors le fond, amenant le mur blanc à réapparaître, ou plutôt à apparaître comme fond: le trait sépare, en fait, deux fonds, deux manières de faire fond, l'une qui entoure, l'autre qui creuse, l'une qui enveloppe l'ancienne présence, l'autre qui crie la nouvelle absence.

Pour la jeune fille, la ligne d'ombre sur le mur est une caresse qui sommeille.

\section{LES MÉTAMORPHOSES DU MIROIR: À FLEUR DE PEAU}

Au livre II de son traité Della Pittura, Alberti fournit une autre origine de la peinture: lâchant cette fois l'ombre pour la proie, il voit dans Narcisse un mythe plus exact que celui de la jeune fille du potier et, dans la mort, une vertu plus prolifique de la mise en figure que celle de l'ombre. Alberti relève, certes, les jugements des Anciens sur la peinture et l'ombre, mais c'est pour écarter rapidement l'histoire (même fabuleuse) au profit d'un examen critique: l'origine de la peinture devient une fable théorique plus instructive que les légendes empiriquement probables.

Ce second livre est chargé, dès l'abord, de justifier les pratiques picturales, de leur donner une assise sociale, voire une légitimité transcendante, car la peinture possède «une force tout à fait divine qui lui permet non seulement de rendre présents, comme on le dit de l'amitié, ceux qui sont absents, mais aussi de montrer après plusieurs siècles les morts aux vivants» ${ }^{7}$. La peinture est un lien: dans le temple protégé de son petit espace, elle recueille la vivante présence des disparus, comme pour une religion du temps commun plus encore que de l'espace social. C'est en quoi Alberti souligne son effet puissant pour aiguillonner la piété des gens : respect du portrait du roi qui est le roi même (comme dans l'exemple de Cassandre tremblant devant l'image d'Alexandre, que rapporte Alberti après Plutarque ${ }^{8}$ ), ou don fait aux mortels d'admirer les dieux.

À quoi repérer la valeur éminente de la peinture? À ce qu'elle devient impayable. Faute de pouvoir trouver un juste prix à ses productions, Zeuxis les donnait. Aussi souverain que le dieu qui donne la vie, le peintre exemplaire donne ses œuvres. Au point que le roi ou le dieu que l'on vénère dans la peinture semble s'effacer derrière sa pure surface, disparaître sous ses rutilantes apparences; c'est le portrait luimême qui fait l'objet de la vénération, métamorphosant le peintre en Créateur, dès lors qu'il se distingue "comme un autre dieu parmi les mortels».

La peinture ne saurait donc être fille de potier sans excéder cette appartenance: tous les autres arts relevaient autrefois de l'artisanat, «seul le peintre n'était pas compté au nombre des artisans " ${ }^{9}$. Et là vient le mythe, un mythe plein d'à-propos (si le latin dit simplement qu'il est "parfaitement approprié», la version italienne que compose aussi Alberti lui donne un tour plus énergique: il vient à-propos - viene a proposito):

C'est pourquoi j'ai l'habitude de dire à mes amis que l'inventeur de la peinture, selon la formule des poètes, a dî être ce Narcisse qui fut changé en fleur car, s'il est vrai que la peinture est la fleur de tous les arts, alors la fable de Narcisse convient parfaitement (perapta erit) à la peinture. La peinture est-elle autre chose que l'art d'embrasser ainsi la surface d'une fontaine (Quid est enim aliud pingere quam arte superficiem illam fontis amplecti)? (Ibid.)

Sans pinceau, sans tableau, sans fatigue, Narcisse est l'occasion de la peinture ${ }^{10}$. Métamorphosé en fleur, il correspond déjà au caractère de celle-ci: être la fleur de tout art (la peinture est transcendante, cette fois-ci au sens où elle traverse toutes les catégories, à l'instar des transcendantaux comme être, un, etc.). Mais l'analogie court au-delà du jeu de mots, car Narcisse est celui qui, tissé de désir, embrasse la surface d'une eau-miroir. La plus profonde vérité sourd de ce geste exaltant la surface. D'autant que fons peut aussi désigner la "source", au sens propre comme au sens figuré. Embrasser la surface de la fontaine-source serait alors comme embrasser la surface de l'origine, l'origine comme surface et non comme profondeur, voire la surface comme origine et non comme trivialité pelliculaire ${ }^{11}$.

Le mythe d'origine de la peinture, chez Alberti, est aussi un mythe sur la superficialité de l'origine: peut- 
être même rien qu'une blague entre amis, une fantaisie de l'art de converser ${ }^{12}$. Mais une blague dont la superficialité est tout sauf triviale: l'amitié, on s'en souvient, est là dès le départ. Par l'allusion à Cicéron, l'amitié permettait aux absents de demeurer présents, la peinture, elle, ramène les morts à la vie comme elle fait surgir les ombres dans la lumière. Même si c'était l'occasion d'une plaisanterie amicale, la peinture n'en perdrait pas pour autant sa profondeur originelle: avec l'amitié, elle est liaison, couture, repli de l'absent dans la présence, du mort dans le vivant, de ce qui sombre dans ce qui éblouit. Ce n'est plus seulement une ligne, qui sépare et unit, mais une tache, qui amalgame et différencie.

Et pourtant le mythe de Narcisse semble dire le contraire: voilà un vivant qui passe chez les morts, un présent qui se noie au fil du temps. Ce serait sans compter sur la métamorphose. Le mythe compte deux temps qui n'en font qu'un: aucun délai entre le fait d'embrasser la surface et l'immédiat changement en fleur ${ }^{13}$. Narcisse ne coule pas dans le brouillard des eaux, il épouse la surface mobile d'une source. La mort n'apparaît pas, sinon effacée sous la puissance de la transformation et renvoyée à ses profondeurs terrifiantes. L'origine, elle, demeure à la surface; c'est une origine qui surnage, loin d'édifier ou de bâtir, une origine sans rituel commandé et sans espace infini.

La métamorphose est une façon de flouer le temps: on croyait Narcisse noyé, voici qu'il éclôt à la surface de l'eau. Plutôt que l'immobilité d'un mur accueillant le dessin hiératique de la silhouette désirée, voici la fine mobilité des eaux qui change sans cesse ses arrangements floraux ${ }^{14}$ : deux façons de dénier au temps son pouvoir ordinaire. Au temps, sans doute, mais à la mort? La métamorphose originaire ne serait-elle pas une image de la métamorphose mortuaire? Et donc, la peinture, puissance de mort? Mais c'est plutôt la mort qui, ellemême, se trouve métamorphosée par la peinture: d'abord, en ce qu'elle apparaît sous la figure vivante des êtres, comme si ce voile était plus originaire que sa nudité; ensuite en ce qu'elle fait surface, comme si sa platitude devenait plus exacte que sa profondeur. La mort se retourne dans sa tombe d'être ainsi projetée à sa surface: Mallarmé, en rigoureux prêtre des définitions, en avait trouvé la formule lorsque autrefois il annonçait «ce peu profond ruisseau calomnié, la mort». C'est en ce ruisseau que Narcisse s'est à la fois noyé et transformé pour mieux en affirmer le pouvoir de surface.

On a dit que c'était sa propre beauté qui arrêtait Narcisse et le faisait se perdre dans son image sans corps. On s'est trompé, comme le prouvent encore les succès nouveaux de l'autoportrait au crépuscule du Moyen Âge: il ne s'agissait pas de se peindre soimême, mais de ressusciter la fascination des miroirs ${ }^{15}$. Narcisse est amoureux d'un reflet qui le fixe obstinément et non de cet être reflété par les hasards de l'onde ${ }^{16}$. Narcisse n'a le sentiment inouï d'exister que regardé par l'œil de son reflet: devenir à la fois visible et voyant, comme à la fois mort et vivant, noyé à la surface. L'art de la perspective dont Alberti vante les productions inédites est, lui aussi, d'abord technique de miroirs, fascination des reflets et des illusions: dans le premier appareillage de Brunelleschi, des nuages fleurissent dans le lit du ciel que les miroirs en cascades attrapent et figent momentanément ${ }^{17}$. Le miroir est, par excellence, la surface de l'immédiat.

Quand Platon cherche à révoquer l'usage de la mimesis, chez le poète d'abord, puis chez le peintre, il renvoie Glaucon à l'expérience, qui n'est autre qu'un pur sophisme (comme Glaucon lui-même est prêt à le remarquer), par où en un instant, très vite, on peut créer

[...] la terre, le ciel, les dieux, tout ce qui existe dans le ciel et tout ce qui existe sous la terre chez Hadès. [...] Ne vois-tu pas que toimême tu pourrais créer tout cela d'une certaine façon? [...] si tu veux prendre un miroir et le présenter de tous côtés; en moins de rien [takhu, immédiatement] tu feras le soleil et les astres $d u$ ciel, en moins de rien, la terre, en moins de rien, toi-même. 18

S'il est une philosophie de la peinture, c'est la sophistique qui la fournit, et son goût des métamorphoses et sa joie tranquille des apparences et 
sa manière de favoriser le saut dans l'immédiat plutôt que le travail de la médiation.

Avec Narcisse, la peinture est double: d'un côté, elle importe la ressemblance, de l'autre, elle exporte la métamorphose. Il faut la ressemblance pour que surgisse la métamorphose, non parce que celle-là serait antérieure à celle-ci, mais juste au contraire, parce que la ressemblance est une métamorphose poussée à bout, une métamorphose fatiguée (ou, peut-être, la vérité fatiguée de la métamorphose).

À la surface de l'eau pousse un regard. La fontaine ou la source sont des surfaces mobiles, il faut, pourtant, la mince peau de sa surface pour que le visage s'y retrouve, même agité de passions qu'il ne connaît pas ou de rides inattendues. La fable n'évoque pourtant pas la fluctuation des eaux, elles semblent tendues comme un drap, étales comme un miroir d'argent, sans rien derrière ni dessous. Une surface sur laquelle fleurit un visage: mais lequel? Au sommet de son vêtement vert, la fleur était déjà le visage de la plante, au bout de son reflet le visage de Narcisse demeure déjà à fleur d'eau. De quoi, en effet, s'y abîmer, puisque l'abîme ici est ce qui affleure.

La peinture ne cherche pas les profondeurs: c'est un art sans herméneutique (ce qui ne signifie pas sans interprétations). Ses secrets sont d'autant mieux celés qu'ils sont offerts à qui les voit: tout tableau ressemble à la lettre volée, dont parle Edgar Poe, qui n'est jamais mieux cachée que lorsqu'elle est exhibée. Le secret de la fascination de Narcisse tient à ce désir sans secret, ouvert à quiconque y plonge son regard. Au fil de l'eau, le visage postiche devient fleur fétiche, gagnant une autorité que le duel des jours ne pouvait octroyer. Dans la métamorphose, comme dans le remplacement du mythe empiriquement vraisemblable de l'ombre par celui, purement allégorique, de Narcisse, s'instaure à l'épicentre du bouleversement de la répétition le choc de la différence: là où la singularité extrême rejoint l'Idée et l'origine, pour mieux, encore une fois, lier ce que chacun croyait désuni.

La surface n'est pas qu'une surface, elle est aussi un fond. Le blanc qui fonde la peinture n'est pas «le néant blanc, la surface redevenue calme où flottent des déterminations non liées» ${ }^{19}$, mais, à l'inverse, la condition de possibilité de la différence. La surface n'offre pas une limite qui serait tout entière manière de borner la représentation, elle opère, au contraire, comme la matrice où ne cesse de disparaître et de naître la détermination finie: la limitation de la forme devient convergence vers un fondement, évanouissement dans l'infini blanc de l'origine. Non pas détermination finie une fois pour toutes évanouie, mais l'instant où elle vacille au bord de la disparition: l'ombre est le corps sur le point de s'effacer, la fleur est la figure d'un visage en train de disparaitre.

S'il fallait absolument une ontologie spécifique à la peinture, elle s'articulerait à un disparêtre. À l'instar de l'amour (et c'est bien pourquoi ces deux mythes ont affaire au désir), la peinture est la perception de l'infini dans la finitude d'un corps, l'infini de cette détermination finie elle-même. Fleur d'ombre dont le cour bat au rythme de l'infini. Est-ce à dire que l'infini est le fond, mais seulement le fond pour la manifestation de l'identique? L'ombre comme reprise de l'amant, la fleur comme retour du visage embrassé? Ce serait, ainsi que le dit Gilles Deleuze, "confondre le concept propre de la différence avec l'inscription de la différence dans l'identité du concept en général " 20 . Dans l'ombre et la fleur, on oublie, en effet, le mur bien droit et l'eau bien étale, le grain de la surface et les minuscules effondrements ou les infinitésimales montagnes de la fontaine: de sauvages différences ne cessent d'y courir ou d'y flotter. C'est du sein du disparate qu'apparitions et disparitions procèdent. De même, dans la convergence à l'infini, on ne cherche peut-être que le ravissement d'une perspective en point de fuite, là où la convergence des traits qui aimante le regard vers l'infini, non seulement permet le surgissement d'une scène au premier plan, mais surtout sort du tableau selon des lignes de pure divergence. C'est qu'il y a toujours, avec la peinture, une obstination à sortir d'elle-même. Ce n'est pas tant la profondeur que l'on y quête (même si la perspective linéaire en a suscité d'innombrables vocations), mais l'appétit de la surface à nous sauter à la figure. Quand Narcisse tente d'embrasser la surface, ce n'est pas pour 
chercher une profondeur derrière l'image qu'il contemple: il est à lui-même le tableau dont il voudrait sortir, son visage est la toile qui se noie dans l'ailleurs, il meurt non d'orgueil, mais de s'évanouir dans la différence pure qui l'accueille enfin. On a tant fait de Narcisse l'abîme du Moi qu'on n'y discerne plus l'intimité de la métamorphose: c'est de se voir autre qui le fascine, non de se rencontrer lui-même.

Avec Narcisse devenu narcisse, la plante est toute visage, comme la peinture toute portrait - même s'il s'agit de peindre une rose séchée ou un lit bêtement vide. L'ovale vénitien d'un visage sait se glisser, aux yeux d'Alberti, sous la figure grecque de Narcisse, comme une courtisane juive pouvait devenir le modèle d'un ange. La peinture, malgré les imaginations théoriques de certains, demeure à mille lieues de toute physiognomonie, car elle ne désire jamais relier des surfaces à des profondeurs, mais des apparences à d'autres apparences.

\section{L'EMPREINTE ET L'ÉTOFFE: LE TISSU DE LA PEAU}

Entre Pline et Alberti, occupés de peinture, une crise majeure des images a eu lieu sur le versant oriental de la chrétienté: l'iconoclasme. C'est parce qu'il existe un réel pouvoir des images que l'on peut trouver autant de plaisir à les détruire. Du coup, bienvenu est tout récit venant conforter ceux qui défendent le droit à représenter le Seigneur et la possibilité de vénérer les images (mais non de les adorer: ce serait alors verser dans l'idolâtrie).

Dans son Histoire ecclésiastique, Eusèbe de Césarée, au IVe siècle, avait évoqué le destin d'Abgar, roi d'Édesse, en conflit avec Hérode et avec les Romains: prenant connaissance des miracles de Jésus, Abgar lui aurait demandé de venir le guérir des maux qui le tourmentaient, et le Christ aurait répondu qu'il lui enverrait un de ses disciples ${ }^{21}$. Un siècle plus tard, on trouve, dans la Doctrine Addaï du pseudo-Labubna et dans l'Histoire d'Arménie de Moïse de Khorène, une commune adjonction à la légende: en plus de la lettre du Christ, Abgar recevrait aussi un portrait qu'en aurait fait Anan, son messager ${ }^{22}$. Mais, avec la Chronique syriaque du pseudo-Zacharie de Mitylène et, surtout, avec l'Histoire ecclésiastique d'Evagrius, à la fin du VIe siècle, le portrait du Christ est devenu la proie d'une légende tout autre, en avance sur son fameux linceul de Turin: plutôt que d'écrire une lettre et de se faire remplacer par un apôtre, il trempe un linge dans l'eau, le passe sur son visage et le donne à Anan l'empreinte de son visage est demeurée inscrite sur l'étoffe, image de Dieu au double sens du terme: faite par Dieu et reflet de Dieu (theoteukton eikona) ${ }^{23}$. Avant de s'installer enfin dans le saint suaire en passant à l'Occident, le linge sacré portant l'image du Christ servira à Jean Damascène, cet ancien fonctionnaire des impôts devenu moine, pour révoquer les arguments des iconoclastes, le temps de deux versions:

Une vieille tradition est parvenue jusqu'à nous: Abgar, prince

d'Édesse, entendit parler du Seigneur et s'enflamma d'amour pour Dieu; il envoya des messagers lui demander de le visiter et ordonna, s'il refusait, que l'on traçât son portrait en peinture; en apprenant cela, celui qui sait tout et qui est tout-puissant prit une étoffe et, l'approchant de son visage, imprima dessus sa figure propre. 24

On raconte l'histoire suivante: Abgarus, roi de la ville d'Édesse, avait envoyé un peintre pour faire le portrait du Seigneur et il n'y arrivait pas parce que son visage brillait d'un éclat insoutenable; le Seigneur couvrit son divin visage de son manteau, et son visage se trouva reproduit sur le manteau qu'il envoya à Abgarus qui le demandait. ${ }^{25}$

La première version paraît plus euphorique, plus linéaire aussi: la voix de la tradition redouble celle de la rumeur, toutes deux suscitent un désir de présence qui fait demander au Christ de venir et, s'il ne vient pas, à tout le moins que l'on en fasse le portrait, désir que le Christ comble immédiatement sur la première étoffe venue. C'est une histoire sans heurts où la représentation croît de façon continue à partir de la présence.

Le second récit commence avec le seul désir de la représentation, mais une représentation devenue impossible: comment figurer la transfiguration d'un Dieu? Aussi le Christ voile-t-il son visage devant le peintre, aussi le Christ dérobe-t-il son apparence trop lumineuse, à la fois par charité pour le pauvre homme 
et pour mieux produire son propre portrait sur l'étoffe photosensible: la disparition du visage fait l'apparition du portrait. Non pas, cette fois-ci, étoffe quelconque, mais le manteau même du Christ, le vêtement qui l'enveloppe et qu'il envoie à Abgarus par une synecdoque qui redouble le portrait que porte le manteau. Il faut que l'étoffe apprivoise la lumière et la rende sous forme d'empreinte. Le peintre est désarmé devant le pur éclat, il doit apprendre, par les plis de l'ombre, à donner à la lumière le volume qui lui convient et l'espace où elle prend sens.

La face du Christ ressemble alors à un masque mortuaire, mais sans la lourdeur humaine du plâtre ou sans l'inutile chaleur de la cire: ce qu'elle moule est aussitôt modulation de l'étoffe (car le moulage suppose un arrêt, là où la modulation est un démoulage incessant ${ }^{26}$ ). L'étoffe, à mi-chemin du mur et de l'eau, entre souplesse lumineuse et opacité rassurante; l'étoffe qui se transporte, à la différence du mur qui demeure et qui conserve ou de l'eau qui fuit et qui trompe; l'étoffe, comme une toile non encore tendue, n'est pas une argile que fixerait le potier, c'est une peau inversée, lignes tressées qui couvrent la peau, qui la prolongent, qui la redoublent: œuvre de protection, bien sûr, mais aussi sensualité d'une caresse inapparente qui s'emmêle au fil de l'existence plutôt que de la mort. Ici, le vêtement est la peau de Dieu et son portrait un lumineux tatouage 27 .

Aux yeux de Jean Damascène, l'iconoclasme trouve là sa déraison: la légitimité de la peinture et, en particulier, des images de Dieu vient justement de cet aveuglement détourné, de la sensualité trouvée à la transcendance - non seulement une transcendance rendue sensible comme on rendrait l'invisible visible, mais surtout une transcendance apparaissant dans la sensualité comme on peut saisir la perspective invisible qui structure aussi bien l'étiquette du visible que l'espace invisible: la théologie de l'icône repose en fait sur la croyance en l'Incarnation, parce que Dieu s'est fait homme dans la personne bien déterminée du Christ, en retour l'image, quoique sensible, est manifestation de l'invisible. À cette perspective, on peut donner le nom d'amour.
Qu'il y ait amour d'un homme pour un Dieu et désir de sa présence ou amour d'un Dieu pour un homme et présence de ce désir ne conduit qu'à circuler de façon entêtée sur des trames qui se répètent. Comme la limaille court vers l'aimant, la lumière aveuglante saute dans les plis d'ombre du tissu: don de la lumière à l'ombre, don de Dieu à l'homme, issus du désir même de l'homme pour la lumière et de Dieu pour l'ombre. Le don trouve son origine dans la différence, de même que la différence aperçoit son fondement dans la répétition.

Il n'est pas question dans ces récits, à strictement parler, d'une "origine de la peinture», pourtant l'on sent bien qu'ils développent une troisième dimension propre à la peinture, au moment où c'est un Dieu qui l'inaugure: après l'ombre et sa trace obstinée sur le mur, après le miroir et sa rapide métamorphose, voici l'empreinte et l'apparition d'un au-delà qu'elle autorise.

Ouvrage du désir d'une jeune fille, du hasard d'une source ou de la charité de Dieu, ce n'est jamais le peintre qui est à l'origine de son art, mais une immédiate métamorphose du regard - métamorphose générique du divin au sensible, de l'humain en végétal; figure noire, visage de fleur ou face aveuglante. Ces trois manières, ces trois tropismes recouvrent pourtant le même élan, différemment orchestré: tantôt amour de l'autre, tantôt amour de soi, tantôt amour de Dieu. Et, du coup, apparaissent les trois façons de la peinture, trois origines tressées à fleur de peau, où la métamorphose est première et le déplacement, souverain: ombre, reflet, empreinte. $\mathrm{Ou}$, si l'on préfère les supports, tissu, miroir et mur, tels sont les rets en lesquels le regard est retenu. Le feu du Dieu ne traverse pas les corps soumis des hommes, il se cache et, se cachant, se révèle; le feu des yeux ne traverse pas les miroirs, il rebondit et nous incendie; le feu sombre de l'absence ne traverse pas les ombres pliées sous la lumière, il s'écrase et nous aplatit.

Ces inventions ont donc affaire au désir et à l'absence présente des corps, affaire à la surface aussi et au support, affaire enfin à la trace sur un tissu, au dessin d'ombre ou à la couleur de la peau. La peinture 
invente le visage ici comme profil, là comme fleur, ailleurs comme vêtement; écriture de l'ombre, écriture de l'eau, écriture de la lumière, à chaque fois circonscription d'une mémoire et d'un bouleversement. L'ombre est métamorphose du corps comme le désir est métamorphose du regard. L'ombre n'est pas la négation du corps, mais l'expression de sa différence, la silhouette de son affirmation, de même que la trace sur une étoffe du visage du Christ ne forme pas le contraire du divin, mais son prolongement sensible, ou de même que la fleur ne renonce pas à Narcisse, mais accentue son caractère déjà végétatif, en accord avec le rythme des saisons et les vertus de l'eau.

Avec les traits du contour de l'ombre émerge ce qui capture; avec le visage qu'on prend comme une fleur, la peinture devient cueillette; avec la Sainte Face qui résorbe sa lumière divine dans l'étoffe sans profondeur, apparait ce qui absorbe. La peinture (en conservant au mot ses deux sens) captive.

Reste à savoir en quoi les songes des origines de la peinture disent quelque chose de la peinture: ce qui fonde est différent de ce qu'il fonde, sinon, ce ne serait pas un fondement; de même que ce qui fonde le vrai n'est lui-même ni vrai ni faux. L'origine est un simulacre antérieur aux vérités, une ombre d'avant les visages du vrai et du faux, une éclosion antérieure au regard qui s'y métamorphose, des traces préliminaires à leur inscription photosensible.

Pourtant, que cherche-t-on dans l'origine? De quoi innerver et modeler chaque présent qui lui succède: le fondement voudrait que tout instant devienne son miroir, l'ombre portée de son grand corps, l'étoffe imbibée de son visage. Mais si l'on cherche moins dans le miroir la ressemblance que le fait même de voir (voire la métamorphose), si l'on trouve moins dans l'ombre la sombre copie que le destin de chaque présence, si l'on découvre moins dans l'étoffe le portrait d'un Dieu que la singularité d'une lumière, alors l'origine est moins fondement que source.

Tel est ce que nous apprennent ces versions de la peinture inventée, versions auxquelles je me permettrais d'ajouter un rêve - qui fut d'ailleurs un cauchemar (datant des années où j'étais Fellow de
King's College à Cambridge). J'étais dans un bureau, la secrétaire du Domus Bursar, celle donc qui s'occupe des bâtiments et des murs que possède King's, est assise. Je m'en vais, déjà j'ai ouvert la porte et, comme Orphée, je fais la faute de me retourner. Je la vois lentement, mais avec violence, projetée sur le mur derrière elle, elle s'y incruste, devenue maintenant tout entière marron sur le fond du mur clair, elle s'y étend et le pénètre comme une tache sombre dont je reconnais distinctement la silhouette et cependant informe: ce n'est pas le fond qui fait surface (comme le disait Hugo de la forme ou Deleuze de la différence), c'est la surface qui aspire ce qui existe. Je respire de plus en plus péniblement comme si j'assistais à une torture incompréhensible, mais ce n'est pas cette femme qui souffre, c'est moi seulement, parce que je la regarde - et que ce regard est une menace. Je souffre tant que je me réveille, même si je n'ose pas ouvrir les yeux. Je pense aussitôt, comme pour me rassurer, c'est un rêve sur l'origine de la peinture. Ce n'est pas une illusion. On se voile les yeux tant qu'on astreint la peinture à l'esthétique de l'illusion, comme si, interloqués par les avanies pigmentaires du caméléon, on oubliait son être de reptile irrévérencieux: jamais il ne s'est agi de rendre l'impression de trois dimensions avec seulement deux. On ne s'amuse pas impunément avec les ombres. La peinture est née de l'aplatissement d'un corps sur une surface.

On a inventé la peinture pour désigner enfin ce qu'était la présence: non le volume d'un corps, non le souvenir d'une origine, non l'assurance d'un signifié, mais le pliage d'un volume dans l'à-plat de la trace. Le dessein de la peinture est de dessiner un présent, au moins un désir de présent, ou, plus exactement, la découverte d'une proximité. Ainsi, avec son visage dans l'eau, ce n'est pas la distance que Narcisse découvre, ni l'écart entre fiction et vérité ou apparence et être, mais, juste au contraire, la proximité. Tout portrait, toute chose peinte nous touche parce qu'elle nous fait toucher au prochain. Tel est aussi le sens théologique de l'histoire du Christ, ou plus largement la valeur éthique de la peinture. Nos visages ne sont pas loin de nous, voilà ce que montre 
le peintre: ils ne nous précèdent pas seulement, ils ne s'écartent pas tant de nous, ils nous reviennent - à la différence extrême de son visage dans un miroir où l'on cherche des beautés, des grâces ou des boutons expurgeables qui nous maintiennent dans la contemplation et l'éloignement de l'identité. Pas de hasard, donc, si l'origine de la peinture respire dans chaque visage, chaque ombre portée, chaque lumière mise à plat, comme un somnambule qui rêverait de son lit. L'origine n'est plus le lointain qui ordonne, mais le proche qui inspire confiance.

\section{N O TES}

1. Pline l'Ancien, 1985: XXXV, V.

2. Voir Varron, De lingua latina, V, 143. On peut remarquer, en sortant du contexte occidental, que l'on retrouve cette ambivalence dans la légende japonaise des origines de la peinture: dans le Nihonshoki (720 apr. J.-C.), les dieux Izanagi et Izanami «enfoncèrent la lance céleste et, en la remuant, sondèrent la profondeur des eaux", à partir de quoi surgirent les îles qui allaient abriter le destin des Japonais; mais dans la version postérieure du Honsho gashi (1678), «le couple divin Izanagi et Izanami, avec la lance céleste sertie de pierres précieuses, traça les limites sur les mers et créa ainsi les îles. Et c'est de là que vient l'art de guider le pinceau qui permet de constituer les formes " (V. Linhartova, 1996: 349 et 311). Les origines de la peinture sont ainsi liées aux fondations des îles japonaises grâce au mouvement qui, à la surface des eaux, trace ou peint (le verbe egaki peut dire les deux activités): de la profondeur recherchée et sondée, l'origine est remontée à la surface.

3. Pline l'Ancien, 1985: XXXV, XLIII.

4. Comme le fait remarquer E. Gombrich, à propos de ce point de la légende, « it may be that portraits in profile were more familiar on coins and early tomb sculpture than in painting (1995: 30).

5. Voir Pline l'Ancien, 1985: XXXV, XXXVI (p. 75). Pour un commentaire scrupuleux de cette question, voir J. Pigeaud, 1995.

6. On conçoit les résistances que ce mythe, lorsqu'on en perçoit la portée, occasionne. Ainsi J. Barrell a fort bien décrit ce qu'il en advient au XVIII siècle, par exemple aux yeux d'un James Barry: «It would be difficult to think of a myth of origin more calculated to degrade the function of painting than this one. It represents the original genre as portraiture, not as history; it valorises servile copying - tracing - at the expense of idealising invention; and it ascribes a priority to the private over the public function of painting" (1992: 162).

7. L. B. Alberti, 1993 (1435): II, 25 (p. 131).
8. Il est intéressant de voir Alberti aller chercher la version antique de Plutarque plutôt que la version chrétienne, et donc canonique, de saint Basile: «Parce que l'image du roi, on l'appelle roi aussi, et qu'il n'y a pas deux rois [...], parce que l'honneur rendu à l'image remonte au prototype, ce que l'image est là par imitation, le Fils l'est ici par nature" (Basile le Grand, 1962: 103 [chap.8]).

9. Alberti, 1993 (1435): 135.

10. Il va sans dire que l'usage (donc aussi la lecture tacite) que fait Alberti du mythe de Narcisse en circonscrit la portée. Nombre d'autres lectures en ont été faites qui n'importent pas à mon propos.

11. Le lien entre origine, surface et peinture est présent, comme on l'a vu, dans la fable japonaise (voir supra, note 2).

12. Le mythe de Narcisse est peut-être aussi propulsé par le néoplatonisme ambiant à Florence ou à Rome, mais le rationalisme, le naturalisme et la conception civique de l'art que manifeste Alberti le font quelque peu résister aux idées et au sérieux d'un Ficin ou d'un Pic de la Mirandole (sur ce sujet, voir A. Blunt, 1966: 40-41).

13. Dans le tableau de Poussin, Écho et Narcisse, on voit en même temps le jeune héros se regarder dans l'eau et le narcisse flotter à la surface, conjoignant ainsi, sur la surface de la toile, l'avant et l'après de la transformation, ou plutôt l'immédiateté de la métamorphose.

14. Félibien retrouve la logique de ce mythe lorsqu'il compose luimême sa propre fable. Cette fois-ci c'est Amour qui cherche son portrait dans les eaux accueillantes des rivières, des lacs et des fontaines, mais lorsque les Nymphes de ces eaux avaient fini le tableau, il était impossible de "le tirer d'entre leurs mains", et sitôt qu'Amour s'éloignait, «elles effaçaient ce qu'elles avaient fait, pour mettre autre chose à la place" (1725: 456 [vol.4])

15. Le miroir est d'ailleurs l'instrument par excellence pour juger de la qualité des peintures, dit un peu plus loin Alberti - pratique qui perdure encore au siècle suivant (Léonard de Vinci, par exemple, s'interroge: « Pourquoi voit-on mieux la peinture dans un miroir que sans lui?» (1987: 151).

16. C'est ce que Pascal saura parfaitement exprimer (même négativement): "Quelle vanité que la peinture qui attire l'admiration par la ressemblance des choses dont on n'admire point les originaux!» (1993: n³7).

17. On sait que l'expérience inaugurale de Brunelleschi consistait à présenter sur un petit panneau le rendu géométrique impeccable du baptistère Saint-Jean que le spectateur devait regarder par un petit trou, tout en tenant le panneau devant lui, mais le ciel et les nuages n'étaient pas peints, puisque Brunelleschi avait simplement installé une mince bande d'argent où ciel et nuages éventuels venaient se refléter. C'est que le ciel et les nuages posent un problème de contours, ils n'ont pas de silhouettes dessinables, pour des raisons inverses: le ciel est tout de surface (tant il est la profondeur même, mais insondable ou inarpentable) et les nuages sont des "corps sans surface" comme les appelle Léonard de Vinci (tant leurs contours sont flous et fluctuants à l'instar de l'eau ou de la fumée). Voir H. Damisch, 1972: 165.

18. Platon, 1982: X, 596c-d.

19. G. Deleuze, 1989: 43

20. Ibid.: 71.

21. Eusèbe de Césarée, 1952: I, XII, 1-22.

22. Moïse de Khorène, 1967: 130-131. Pseudo-Labubna, 1981.

23. Zacharie de Mitylène, 1870: 324-325; Evagrius, 1964: 175, IV, 27. Sur ces récits, ou plus généralement sur les implications théologiques de la querelle, voir A. Desreumaux, 1987: 73-79; M.-J. Mondzain, 1996; C. von Schönborn, 1976.

24. J. Damascène, 1994: 103. 
25. J. Damascène, 1966: IV, XVI.

26. «La circulation du support d'énergie équivaut à un démoulage permanent; un modulateur est un moule temporel continu». G. Simondon, 1964 : 41.

27. On peut remarquer qu'un des points d'affrontement entre iconoclastes et iconodoules touche à la "circonscription" du portrait: pour ceux-là, le Christ, rendu à sa gloire divine, est devenu matériellement incirconscriptible; pour ceux-ci, soit il faut distinguer (comme le recommande saint Nicéphore de Constantinople) entre la graphê, peinture physique des corps, et la perigraphê, circonscription dans l'espace, dans le temps et dans la compréhension qui relève du domaine notionnel (ou, pour prendre un vocabulaire plus tardif, du "transcendantal») et non de l'univers du sensible, soit (comme y engage saint Théodore Studite) on doit admettre que la peinture circonscrit non la nature, mais bien l'" hypostase" de la personne représentée, selon la parole littérale de l'Épître aux Hébreux, I, 3 ( Il est le rayonnement de sa gloire et l'empreinte de son hypostase [upostaseos]»), ce qui est autre que l'empreinte de sa substance (autant la substance est indéterminée, autant l'hypostase suppose le fait même de la détermination): en ce sens, on pourrait y rapporter l'emprise du néoplatonisme et dire que l'icône circonscrit seulement l'ombre de la véritable réalité du Christ, mais Théodore (et plus largement la théologie iconodoule) esquive cette dépréciation néo-platonicienne du monde des images et du sensible lorsqu'il affirme que «l'icône est le témoin fidèle de ce que le Verbe s'est fait semblable aux hommes " (1857, II, 65) - de même, l'ombre que circonscrit la jeune fille du potier est témoignage plus encore que trace, mémoire plutôt qu'histoire.

\section{RÉFÉRENCES BIBLIOG RAPHIQ U ES}

I. Textes anciens

AlberTI, L. B. [1435]: De la peinture: De Pictura, préf. et trad. de J. L. Schefer, intro de S. Deswarte-Rosa, Paris, Macula, 1993.

Basile Le Grand [1962]: Traité de l'Esprit Saint, Paris, Desclée de Brouwer.

EusĖBe DE CÉSARÉE [1952]: Histoire ecclésiastique, I, XII, Paris, Cerf, 122.

Evagrius [1964]: The Ecclesiastical History, s. la dir. de J. Bidez et L.

Parmentier, Amsterdam, Adolf Kakkert.

FÉLIBIEN, A. [1725]: Entretiens sur les vies et les ouvrages des plus excellents peintres, Trévoux.

JeAn DAMASCĖNe [1994]: Discours sur les images, dans Jean Damascène:

Le visage de l'invisible, trad. A.-L. Darras-Woms, Paris, Migne;

[1966] : La Foi orthodoxe, trad. E. Ponsoye, Paris, Migne, IV, XVI. MOÏSE DE KHORĖne [1967]: Histoire d'Arménie, dans W. Cureton, Ancient Syriac Documents Relative to the Earliest Establishment of

Christianity in Edessa and the Neighbouring Countries, Amsterdam, Oriental Press.

PASCAL, B. [1993]: Pensées, s. la dir. de M. Le Guern, Paris, Gallimard. PlATON [1982]: République, trad. E. Chambry, Paris, Les Belles Lettres. Pline L'Ancien, [1985]: Histoire naturelle, trad. J.-M. Croisille, Paris, Les Belles Lettres.

PSEUdD-LABUbNa [1981]: The Teaching of Addaï, s. la dir. de G. Howard, Chicago, Society of Biblical Literature.

SAINT THÉODORE STUdiTE [1857]: Épittres, dans Patrologia cursus, series graeca, II, s. la dir. de J. P. Migne, Paris, Garnier Frères.

VARRON [1954]: De Lingua latina, Paris, Les Belles Lettres.

VINCI, L. de [1987]: Traité de la peinture, trad. et prés. par A. Chastel, Paris, Éd. Berger-Levrault.

ZACHARIE DE MitYLĖNe [1870]: Anecdoton Syriacorum, s. la dir. de J. P. N. Land, Leyde, 324-325 (trad. de F. J. Hamilton, E. W. Brooks, The Syriac Chronicle Known as that of Zachariah of Mitylene, Londres, Methuen, 1899, 320-322).

II. Textes modernes

BARrell, J. [1992]: The Birth of Pandora, and the Division of Knowledge,

London, Macmillan.

BLunT, A. [1966]: La Théorie des arts en Italie de 1450 à 1600, trad. J.

Debouzy, Paris, Gallimard.

Damisch, H. [1972]: Théorie du nuage, Paris, Seuil.

Deleuze, G. [1968] : Différence et Répétition, Paris, P.U.F., 1989.

DesreumauX, A. [1987]: «La doctrine d'Addaï, l'image du Christ et les Monophysites ", dans Nicée II, 787-1987, douze siècles d'images religieuses, s. la dir. de F. Boespflug et N. Lossky, Paris, Cerf, 73-79.

GOMBRICH, E. [1995]: Shadows: The Depiction of Cast Shadows in Western Art, Londres, National Gallery Publications.

LINHARTOVA, V. [1996]: Sur un fond blanc: Écrits japonais sur la peinture du IXe au XIXe siècle, Paris, Le Promeneur.

MondzAIN, M.J. [1996]: Image, icône, économie: Les sources byzantines de l'imaginaire contemporain, Paris, Seuil.

PigEAUD, J. [1995] : «La rêverie de la limite dans la peinture antique», L'Art et le Vivant, Paris, Gallimard.

SCHÖnBORN, C. von [1976]: L'Icône du Christ: Fondements théologiques, Fribourg, Éd. universitaires de Fribourg.

SimONDON, G. [1964]: L'Individu et sa genèse physico-biologique, Paris, P.U.F. 